\title{
Temporal and Spatial Development of Sorghum Anthracnose in Arkansas
}

\author{
Yonghao Li and David O. TeBeest, Department of Plant Pathology, University of Arkansas, Fayetteville, AR 72701
}

\begin{abstract}
Li, Y. H., and TeBeest, D. O. 2009. Temporal and spatial development of sorghum anthracnose in Arkansas. Plant Dis. 93:287-292.

Development of sorghum anthracnose in time and space on susceptible ('BTx623' and 'Pioneer 8313') and resistant ('Cargill 888Y') sorghum (Sorghum bicolor) cultivars was investigated in field plots inoculated with a mixture of three strains (T430-SW, Ar54-SW, and ArSS-1SW) of Colletotrichum sublineolum in 2001 and 2002. After slow development during early growth stages, sorghum anthracnose developed rapidly after flowering. The logistic model provided a good statistical fit for disease progress curves for sorghum anthracnose in Arkansas. Compared to susceptible cultivars, the resistant cultivar had significantly lower values for the standard area under the disease progress curve, a lower upper asymptote, and delayed epidemic onset. However, there were no significant differences in infection rate and the time of the inflection points, the times at which the disease proportion equaled values of one-half of upper asymptotes, among the three cultivars. The negative exponential model more closely described disease gradients of sorghum anthracnose than the inverse power law model. There were significant differences in intercepts but not in slopes and half-distance between the resistant and susceptible cultivars. The slopes of disease gradients were significantly lower at 78 days after inoculation (DAI) than at 57 DAI on the three cultivars. The results could provide epidemiological components of sorghum anthracnose to help evaluate sorghum cultivars and breeding lines under field conditions.
\end{abstract}

Sorghum (Sorghum bicolor (L.) Moench) is one of the major food crops, particularly in areas of high temperature and low rainfall, and is ranked fifth in importance among the world's cereals (17). Sorghum anthracnose, caused by Colletotrichum sublineolum Hann. Kabát et Bub. (syn. C. graminicola (Ces.) G.W. Wils.), remains one of the most important diseases and is crop-limiting in some sorghum production areas $(12,29)$. Estimated grain sorghum losses caused by anthracnose were about $50 \%$ on susceptible cultivars (28-30). Sorghum anthracnose has been an important disease in Arkansas since 1988 (9). Moore et al. (19) recently described the existence of several additional pathotypes of $C$. sublineolum that were virulent to most differentials in $\mathrm{Ar}$ kansas.

Sorghum residues on the soil surface have an important role as a source of primary inoculum $(1,6,7,13)$. Infected seeds with visible acervuli are considered to be another important source of primary inocu$\operatorname{lum}(5,26)$. Conidia produced on diseased plants serve as secondary inoculum within

Corresponding author: D. O. TeBeest

E-mail: dtebeest@uark.edu

Current address of Yonghao Li: Department of Entomology and Plant Pathology, University of Tennessee, Knoxville 37996-4560.

Accepted for publication 25 November 2008.

doi:10.1094/PDIS-93-3-0287

(c) 2009 The American Phytopathological Society fields. Secondary inoculum is dispersed by rain-splash to susceptible host tissues. The disease spreads from foci up to $3.25 \mathrm{~m}$ at the 13- to 14-leaf stage and up to $9.75 \mathrm{~m}$ at physiological maturity (23). The disease is most severe during extended periods of cloudy, warm, humid, and wet weather, especially when these conditions occur during the early grain-filling period (12). Ngugi et al. (20) reported that sorghum anthracnose developed slowly before anthesis and increased rapidly during the grain-filling stage in Africa, and disease progress curves were best described by a logistic model.

One purpose for studying plant disease epidemiology is to understand dynamics of diseases in time and space as the result of the interactions of host, pathogen, and environment (4). The epidemic onset, infection rate, time at inflection point, final amount of disease, and area under disease progress curve (AUDPC) have been used to compare and evaluate disease management strategies including the use of fungicides, cultural practices, or resistant cultivars $(15,20,21,25,27,28,34)$. In this regard, Ngugi et al. (20) reported that there were significant differences in time of inflection point, disease onset, absolute rate, disease severity, and upper asymptote values for sorghum anthracnose epidemics caused by unknown field isolates among sorghum cultivars in Kenya. AUDPC values have also been used to compare resistance to sorghum anthracnose among sorghum genotypes (15). Quantitative analyses of disease gradient were used to investigate inoculum sources, determine the spatial effects of host resistance, test models of disease spread, and identify plot sizes and spacing that would decrease interference to acceptable levels in field experiments $(18,24)$. Similar studies have not been conducted in Arkansas or the United States.

The objectives of this study were to quantify disease progress and the dispersal gradients of sorghum anthracnose in Arkansas caused by three isolates from selected pathotypes of $C$. sublineolum on three specific grain sorghum hybrids or breeding lines known to have different levels of resistance to the three pathotypes of the pathogen and to evaluate selected parameters for comparison of host resistance in the field.

\section{MATERIALS AND METHODS}

Experimental design. Field plots were established in 2001 and 2002 at the Main Experiment Station of the University of Arkansas at Fayetteville. Three sorghum cultivars, 'BTx623', 'Pioneer 8313', and 'Cargill 888Y', were planted in a randomized complete block design with four replications. Each plot consisted of ten 5-mlong rows with 1-m space between rows. Two rows of sorghum-sudan grass were planted between blocks and on the borders of the fields to reduce interplot interference. Approximately 140 sorghum seeds were planted in each row within plots on 9 May 2001 and 7 May 2002, respectively. To control weeds in the field plots, the herbicide atrazine was applied prior to emergence at a rate of $1 \mathrm{lb} / \mathrm{A}$. Seedlings were thinned to 10 to $15 \mathrm{~cm}$ apart at the four- to five-leaf stage, and any surviving weeds in the plots were removed by hand.

Inoculation. Three strains (T-430SW, Ar54-SW, and ArSS-1-SW) of C. sublineolum were used in the experiments. These strains were deposited in cryostorage facilities in the Department of Plant Pathology at the University of Arkansas, Fayetteville, by Ware (31). T-430SW and ArSS$1 \mathrm{SW}$ were both identified as pathotype 4, while Ar54-SW was shown to be pathotype 0 as described by Moore et al. (19), since it was not virulent to the eight differential lines previously used to describe pathotypes $(5,9)$. The virulence of each of the isolates was re-examined at the conclusion of this study, and all isolates were found to be consistent with the above descriptions. All isolates were deposited in the collection in the Department of Plant Pathology at the conclusion of this study and after pathotyping.

Grain inoculum of the three strains was prepared by washing sorghum seeds in 
water to remove dust and residue, dispersing the seeds into 1-liter flasks (400 $\mathrm{ml}$ of seed per flask), and adding $25 \mathrm{ml}$ of water per flask. Flasks containing sorghum seeds were autoclaved twice at 48$\mathrm{h}$ intervals, and then inoculated separately with five culture pieces $\left(0.25 \mathrm{~cm}^{2}\right)$ taken from 20-day-old cultures of the three strains grown on yeast-potassium-starchsulfate (YPSS) agar medium (4 $\mathrm{g}$ of yeast extract, $1 \mathrm{~g}$ of $\mathrm{K}_{2} \mathrm{HPO}_{4}, 15 \mathrm{~g}$ of soluble starch, $0.5 \mathrm{~g}$ of $\mathrm{MgSO}_{4}$, and $16 \mathrm{~g}$ of agar per liter). Flasks were incubated at room temperature and shaken by hand at 2-day intervals beginning 1 week after inoculation. After 4 weeks, colonized sorghum seeds were removed from flasks, air-dried at room temperature for a week, and stored in paper envelopes at room temperature.

To inoculate the field plots, six colonized seeds ( 2 seeds for each strain) were placed in the leaf whorl of all plants in row two of each plot at the elongation stage on 3 and 11 July in 2001 and 2002, respectively. These inoculated plants served as the only source of inoculum in each plot. After inoculation, plots were irrigated with an overhead sprinkler irrigation system immediately for $2 \mathrm{~h}$ and irrigated for $2 \mathrm{~h}$ twice a week whenever it did not rain during the week.

Disease assessment. To analyze disease progress over time, nine randomly selected plants in row three $(1 \mathrm{~m}$ from the inoculated row) in each plot were marked at 11 days after inoculation (DAI), and the disease severity on five leaves downward from the flag leaf of each plant was assessed using a 0 to 6 scale $(0=$ no disease; $0.1=$ less than $0.5 \%$ of diseased area; 0.5 $=0.5$ to $1 \% ; 1=1$ to $5 \% ; 2=5$ to $15 \% ; 3$ $=15$ to $30 \% ; 4=30$ to $50 \% ; 5=50$ to $75 \%$; and $6=75$ to $100 \%$ ) at 5- to 10 -day intervals. Assessments were made 11 times from July to September in both years. For the precision assessment of disease severities, a single rater collected all data during the entire experiment. Before the disease progress analysis, the data were transformed to the midpoint of disease severity for each scale and averaged for each cultivar at each sampling date.

To analyze disease gradients, disease severity on nine randomly selected plants in each of rows $2,3,5,7$, and 9 (equivalent to $0,1,3,5$, and $7 \mathrm{~m}$ from the inoculum source, respectively) were assessed and transformed as described above at 57 and 78 DAI in both years.

Data analysis. Disease proportion of sorghum anthracnose was analyzed by cultivar and year over time using linearized forms of the exponential $\left[\ln (y)=\ln \left(y_{0}\right)+\right.$ $r t]$, logistic $\left\{\ln [y /(1-y)]=\ln \left[y_{0} /\left(1-y_{0}\right)\right]+\right.$ $r t\}$, and Gompertz $\{-\ln [-\ln (y)]=-\ln [-$ $\left.\left.\ln \left(y_{0}\right)\right]+r t\right\}$ models (4) in order to describe temporal disease development. The coefficient of determination $\left(R^{2}\right)$, the mean square error (MSE), and patterns of resid- ual plots were used to evaluate the appropriateness of the models to describe the observed data. Although the models tested were similar to each other in their description of disease progress, all were less appropriate than the logistic model that was chosen for estimation of epidemic parameters.

The least-squares program for nonlinear model (NLIN) procedure of SAS software (Version 8, SAS Institute Inc., Cary, NC) was used to obtain estimates of parameters from the nonlinear form of the logistic model (equation 1).

$$
y=\frac{k}{1+\left(\frac{k-y_{0}}{y_{0}}\right) e^{-r t}}
$$

In this equation, $y$ is the disease proportion at time $t ; k$ is the upper asymptote of the disease progress curve; $y_{0}$ is the initial disease proportion; $r$ is the apparent infection rate; and $t$ is the time of disease assessment in DAI.

The time at $y=k / 2$, or inflection point $\left(t_{i p}\right)$, and the time at $y=0.02$, or epidemic onset $\left(t_{0.02}\right)$, were estimated with the three parameters $\left(k, y_{0}\right.$, and $\left.r\right)$ using equations 2 and 3, respectively, which were derived from equation 1 .

$t_{\text {ip }}=\frac{\ln \left(\left(k-y_{0}\right) / y_{0}\right)}{r}$

$t_{0.02}=\frac{\ln \left[0.02\left(k-y_{0}\right) / y_{0}(k-0.02)\right]}{r}$

To compare means of estimated $t_{i p}$ and $t_{0.02}$ among cultivars, variances of $t_{i p}$ and $t_{0.02}$ were approximated by equations 4 and 5 .

$$
\begin{aligned}
& s^{2}\left(t_{i p}\right)=E^{2} s^{2}(k)+\frac{t_{i p}^{2} s^{2}(r)}{r^{2}}+F^{2} s^{2}\left(y_{0}\right)- \\
& \frac{2 E t_{i p} s(k, r)}{r}+2 E F s\left(k, y_{0}\right)-\frac{2 F t_{i p} s\left(r, y_{0}\right)}{r} \\
& s^{2}\left(t_{0.02}\right)=G^{2} s^{2}(k)+\frac{t_{0.02}^{2} s^{2}(r)}{r^{2}}+F^{2} s^{2}\left(y_{0}\right)- \\
& \frac{2 G t_{0.02} s(k, r)}{r}+2 F G s\left(k, y_{0}\right)-\frac{2 F t_{0.02} s\left(r, y_{0}\right)}{r}
\end{aligned}
$$

In equations 4 and 5,

$$
\begin{aligned}
& E=\frac{2\left(y_{0}-k / 2\right)}{r k\left(k-y_{0}\right)}, \quad F=\frac{-k}{r y_{0}\left(k-y_{0}\right)}, \\
& G=\frac{y_{0}-0.02}{r(k-0.02)\left(k-y_{0}\right)} .
\end{aligned}
$$

$s^{2}(k), s^{2}(r)$, and $s^{2}\left(y_{0}\right)$ are variances of estimates $k, r$, and $y_{0}$, respectively; and $s(k, r)$ is the covariance of estimates $k$ and $r ; s\left(k, y_{0}\right)$ is the covariance of estimates $k$ and $y_{0}$; and $s\left(r, y_{0}\right)$ is the covariance of estimates $r$ and $y_{0}$. Comparisons for estimates among cultivars in each year were conducted using the two-sample $t$ test at $P=0.05$.

The standard area under the disease progress curve (sAUDPC) was calculated for each plot using equation 6 , in which
AUDPC (4) was estimated between the observation days, and

$$
s A U D P C=\sum_{i}^{n-1}\left(\frac{y_{i}+y_{i+1}}{2}\right)\left(t_{i+1}-t_{i}\right) /\left(t_{n}-t_{1}\right)
$$

in which $t_{i}$ is the time at $i$ th observation, $t_{i+1}$ is the time at $(i+1)$ th observation, $y_{i}$ is the disease severity $(\%)$ at $i$ th observation, $y_{i+1}$ is the disease severity at $(i+1)$ th observation, $t_{1}$ is the time at the first observation, and $t_{n}$ is the time at the last observation. The effects of blocks and cultivars on sAUDPC were determined by analysis of variance (ANOVA) using the GLM procedure of SAS software, and mean separations for sAUDPC were conducted using the least significant difference (LSD) test at $P=0.05$.

For analysis of disease development in space, mean disease proportion values at each distance for each cultivar were transformed using the negative exponential (equation 7) and inverse power law (equation 8) models (4), respectively.

$\ln (y)=\ln (a)-b d$

$\ln (y)=\ln (A)-b \ln (d)$

in which $y$ is the disease proportion at distance $d, d$ is the distance from the source of inoculum, $b$ is the slope of the disease gradient, $e$ is the base of natural logarithms, $a$ is the disease proportion at the source point, and $A$ is the disease proportion at one unit of distance from the source (equation 2). $R^{2}$, MSE, patterns of residual plots, and the standard errors associated with the parameter estimates were used to evaluate the appropriateness of the models to describe the observed data. After selection of the most appropriate model, fit was tested for a series of models using the average disease proportion for each cultivar, which allowed differentiation of intercepts and slopes for each of the six dates by cultivar combinations. Depending on the results of the initial fit, additional models were fitted which put restrictions on the slopes and the intercepts. Final estimated parameters were compared using single degree of freedom contrasts. The half distance $\left(d_{50 \%}\right)$, at which the disease proportion declined to $50 \%$ of that at the source $(y=a / 2)$, from the negative exponential model was estimated using equation 9, which was derived from equation 7 .

$d_{50 \%}=0.6931 / b$

To compare $d_{50 \%}$ among cultivars, variances for $d_{50 \%}$ were approximated by equation 10:

$s^{2}\left(d_{50 \%}\right)=\left(\frac{0.6931}{b^{2}}\right)^{2} s^{2}(b)$

in which, $s^{2}(b)$ is the variance of estimated slope of disease gradient. The differences in $d_{50 \%}$ among cultivars and sampling dates were compared using the two-sample $t$ test at $P=0.05$. 


\section{RESULTS}

Disease progress curves, plotted as disease proportion against days after inoculation, showed that epidemics of sorghum anthracnose in the rows that were adjacent to inoculated rows began about 40 to 45
DAI (flowering stage in mid-August), although lesions appeared in the inoculated rows at 10 DAI. The disease developed rapidly from 50 to $80 \mathrm{DAI}$ (from the end of flowering to grain-filling stages) on BTx623 and Pioneer 8313, while the dis-
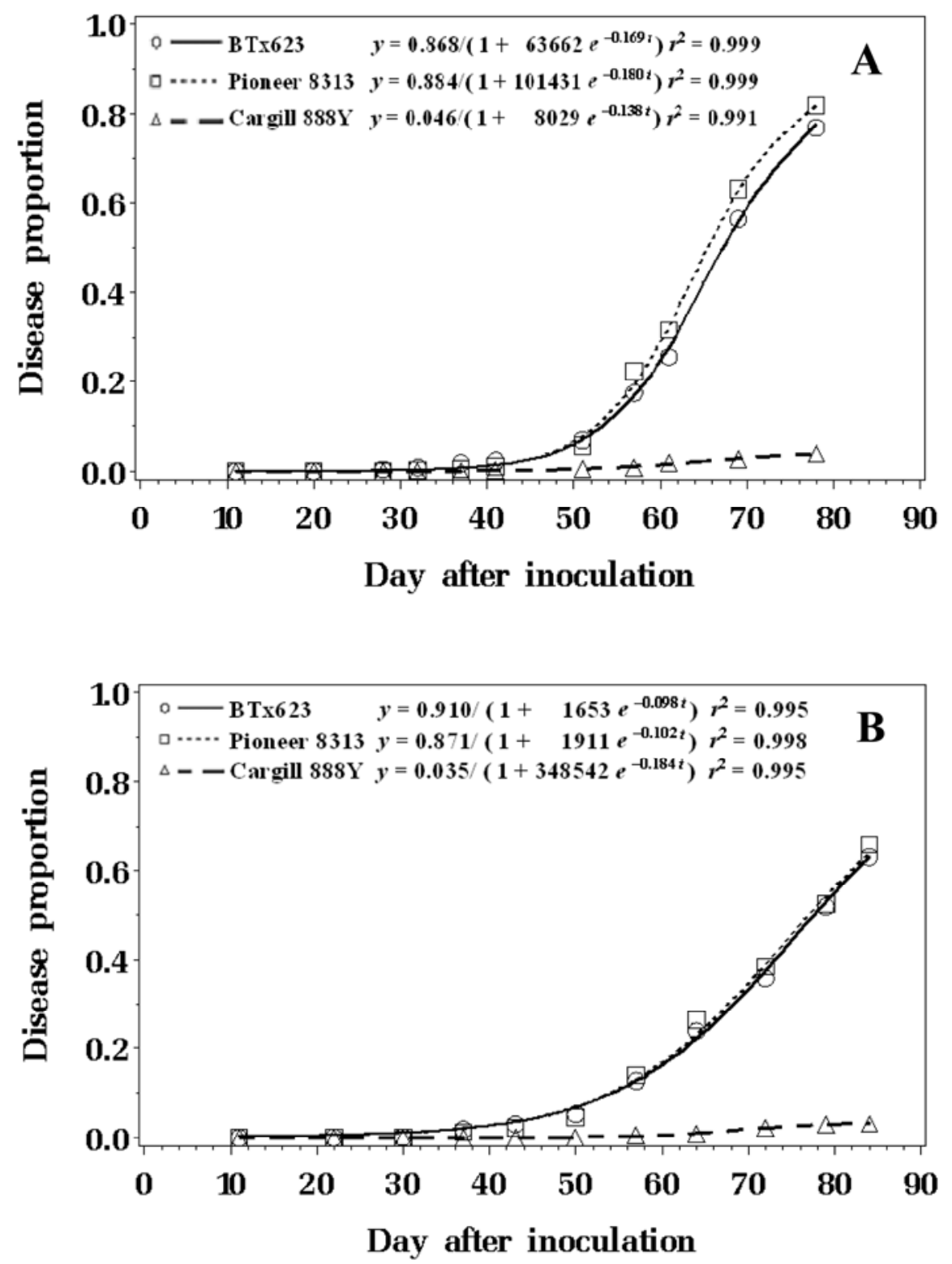

Fig. 1. Disease progress of sorghum anthracnose estimated using the nonlinearized logistic model with average disease proportion of four plots on three cultivars $1 \mathrm{~m}$ from rows inoculated with a mixture of the three strains T430-SW, AR54-sw, and ArSS-1SW on 3 and 11 July in 2001 (A) and 2002 (B), respectively. Symbols and smooth lines represent observed values of disease proportions and best fit of the logistic model, respectively.

ease severity on Cargill 888Y remained low during the entire season (Fig. 1). Based on $R^{2}$, MSE, and patterns of residual plots, the logistic model provided excellent approximations for the disease progress data. Fitted curves closely resembled plots of the actual data and the approximate coefficient of determination $\left(R^{2}\right)$ values were above 0.99 (Fig. 1).

The effects of cultivars on values of $k$ and $t_{0.02}$ in disease progress were constant in 2 years (Table 1). The susceptible BTx623 and Pioneer 8313, had significantly higher values of $k$ and earlier $t_{0.02}$ than the resistant Cargill 888Y. No significant differences in $k$ and $t_{0.02}$ were found between the two susceptible cultivars (Table 1). However, the effects of cultivars on $r$ and $t_{i p}$ as estimated by the logistic model were not constant and were not related to observed differences in resistance among the cultivars in 2 years. In 2001, $r$ was not significantly different among cultivars. Infection rate for the resistant cultivar was significantly higher than for the susceptible cultivars in 2002. The $t_{i p}$ for the resistant cultivar was significantly later than for Pioneer 8313 , but not significantly different from that for BTx623 in 2001. However, Cargill $888 \mathrm{Y}$ had significantly earlier $t_{i p}$ than BTx623 and Pioneer 8313 in 2002 (Table 1).

The results of ANOVA showed that cultivars significantly affected the sAUDPC values of sorghum anthracnose in both $2001(P=0.0215)$ and $2002(P<0.0001)$. The results of mean comparisons showed that sAUDPC values on BTx623 or Pioneer 8313 were 20 -fold greater than those on Cargill 888Y. There were no significant differences in sAUDPC between BTx623 and Pioneer 8313 in both years (Table 2).

In results of linear regression statistics using $R^{2}$ and MSE, the negative exponential model was more appropriate in describing disease gradients of sorghum anthracnose than the inverse power law model in both years. Results of analyses using the negative exponential model showed that intercepts depended on both dates $(P<0.0001$ in 2001 and $P=0.0103$ in 2002) and cultivars $(P<0.0001$ in both years), but not on the observation date and cultivar interaction $(P=0.1093$ in 2001 and $P=0.5252$ in 2002). Slopes depended on dates $(P<0.0001$ in both years $)$ but not on cultivars $(P=0.0429$ in 2001 and $P=$

Table 1. Comparisons for asymptote $(k)$, apparent infection rate $(r)$, time of inflection point $\left(t_{i p}\right.$, day), and time of epidemic onset $\left(t_{0.02}\right.$, day) estimated from nonlinear regression of disease progress of sorghum anthracnose with the logistic model for three cultivars in 2001 and 2002

\begin{tabular}{lllclc}
\hline Year & Cultivar & $\boldsymbol{k}(\mathbf{S E})^{\mathbf{y}}$ & $\boldsymbol{r}(\mathbf{S E})$ & $\boldsymbol{t}_{\boldsymbol{i} \boldsymbol{p}}(\mathbf{S E})$ & \multicolumn{1}{c}{$\boldsymbol{t}_{\mathbf{0 . 0 2}}(\mathbf{S E})$} \\
\hline 2001 & BTx623 & $0.868(0.062) \mathrm{a}$ & $0.169(0.021) \mathrm{a}$ & $65.7(0.561) \mathrm{a}$ & $43.6(2.286) \mathrm{b}$ \\
& Pioneer 8313 & $0.884(0.020) \mathrm{a}$ & $0.180(0.009) \mathrm{a}$ & $63.9(0.213) \mathrm{b}$ & $43.0(0.818) \mathrm{b}$ \\
& Cargill 888Y & $0.046(0.005) \mathrm{b}$ & $0.138(0.024) \mathrm{a}$ & $65.0(0.868) \mathrm{a}$ & $63.2(0.805) \mathrm{a}$ \\
& BTx623 & $0.886(0.071) \mathrm{a}$ & $0.102(0.007) \mathrm{b}$ & $75.0(0.356) \mathrm{a}$ & $38.1(1.718) \mathrm{b}$ \\
& Pioneer 8313 & $0.864(0.099) \mathrm{a}$ & $0.105(0.012) \mathrm{b}$ & $73.3(0.638) \mathrm{b}$ & $37.7(2.784) \mathrm{b}$ \\
& Cargill 888Y & $0.034(0.002) \mathrm{b}$ & $0.190(0.021) \mathrm{a}$ & $69.1(0.504) \mathrm{c}$ & $70.8(0.533) \mathrm{a}$ \\
\hline
\end{tabular}

${ }^{y}$ All values enclosed in parentheses are standard errors (SE).

${ }^{\mathrm{z}}$ Means followed by same letter within a column for each year were not significantly different from each other at $P=0.05$ according to two sample $t$-tests. 
0.0729 in 2002) or their interactions $(P=$ 0.3028 in 2001 and $P=0.2006$ in 2002) (Fig. 2). The results of comparisons for estimates from disease gradient models using linear contrasts (Table 3 ) showed that cultivars BTx623 and Pioneer 8313 had significantly higher intercept values than Cargill $888 \mathrm{Y}$ in two sampling dates and 2 years, and the values of intercepts for all cultivars at 78 DAI were significantly higher than those at 57 DAI in both years. The slopes and half-distance values for all cultivars at 78 DAI were significantly shallower and shorter than those at 57 DAI, respectively, in both years (Table 3 ).

\section{DISCUSSION}

These results are the first report of temporal development of sorghum anthracnose in Arkansas and in the United States. The results are similar to the results reported by Ngugi et al. (20) on sorghum anthracnose in Kenya. A significant difference between our research and that of Ngugi et al. (20) is that we described the development of anthracnose on three hybrids with known levels of resistance and used three isolates from known pathotypes. In the present study, sorghum anthracnose increased rapidly from 40 DAI (mid-August) in rows that were adjacent to inoculated rows. Although there were lower final disease severities in 2002 than in 2001, the disease progress curves for the three cultivars fit the logistic model well in both years.

The upper asymptote $(k)$ is the estimated capability of disease epidemics, and the $k$ value is the result of the interaction of host resistance, pathogen variance, and environment (33). The negative relationship of upper asymptote and disease resistance has been previously reported for sorghum anthracnose (20). In the present study, Cargill $888 \mathrm{Y}$ had significantly lower $k$ values estimated from the logistic model than BTx623 or Pioneer 8313 did, suggesting that reducing epidemic capability may be an important trait of the resistance in Cargill 888Y.

Epidemic onset is an important parameter to compare the overall effects of host resistance on disease development for

Table 2. Multiple comparisons for means of standard area under the disease progress curve of sorghum anthracnose among cultivars in 2001 and 2002

\begin{tabular}{lrr}
\hline & \multicolumn{2}{c}{ Mean $^{\mathbf{y}, \mathbf{z}}$} \\
\cline { 2 - 3 } Cultivar & \multicolumn{1}{c}{$\mathbf{2 0 0 1}$} & \multicolumn{1}{c}{$\mathbf{2 0 0 2}$} \\
\hline BTx623 & $12.244 \mathrm{a}$ & $14.896 \mathrm{a}$ \\
Pioneer 8313 & $16.736 \mathrm{a}$ & $15.564 \mathrm{a}$ \\
Cargill 888Y & $0.753 \mathrm{~b}$ & $0.727 \mathrm{~b}$ \\
\hline
\end{tabular}

${ }^{y}$ All data represent the average of four replications for 'BTx623' and 'Pioneer 8313', and three replications for 'Cargill 888Y' in 2001.

${ }^{z}$ Means followed by same letter within a column were not significantly different from each other at $P=0.05$ according to least significant difference.
Cylindrocladium black rot (8) and sorghum anthracnose (20). In the present study, the delayed epidemic onset (about $40 \mathrm{DAI}$ ) in rows that were adjacent to inoculated rows suggests that several disease cycles were needed to accumulate a sufficient amount of inoculum for disease dispersal. An additional and significant delay of the epidemic onset on Cargill 888Y (63.2 and 70.8 DAI) indicates that more time is needed for the production of a sufficient amount of inoculum on the resistant cultivar than on susceptible cultivars. These results also suggest that the $t_{0.02}$ value is a useful epidemic characteristic in evaluating the level of resistance to sorghum anthracnose in fields as previously showed by Ngugi et al. (20).
Infection rate is considered to be a useful parameter for quantitative assessments of the effects of treatments and rapid comparisons of epidemics (34). But infection rate in the logistic model can be less sensitive than other parameters for some treatments $(14,20)$. Campbell (3) stated that it is not possible to simply compare rate parameter values if estimates of $k$ vary among individual disease progress curves. In the present study, the different results of comparisons of the estimated $r$ from the logistic model among cultivars could be due to large differences in upper asymptotes since they were about 20 times greater for susceptible cultivars than for the resistant cultivar. Since the infection rate is the constant of proportionality rela-
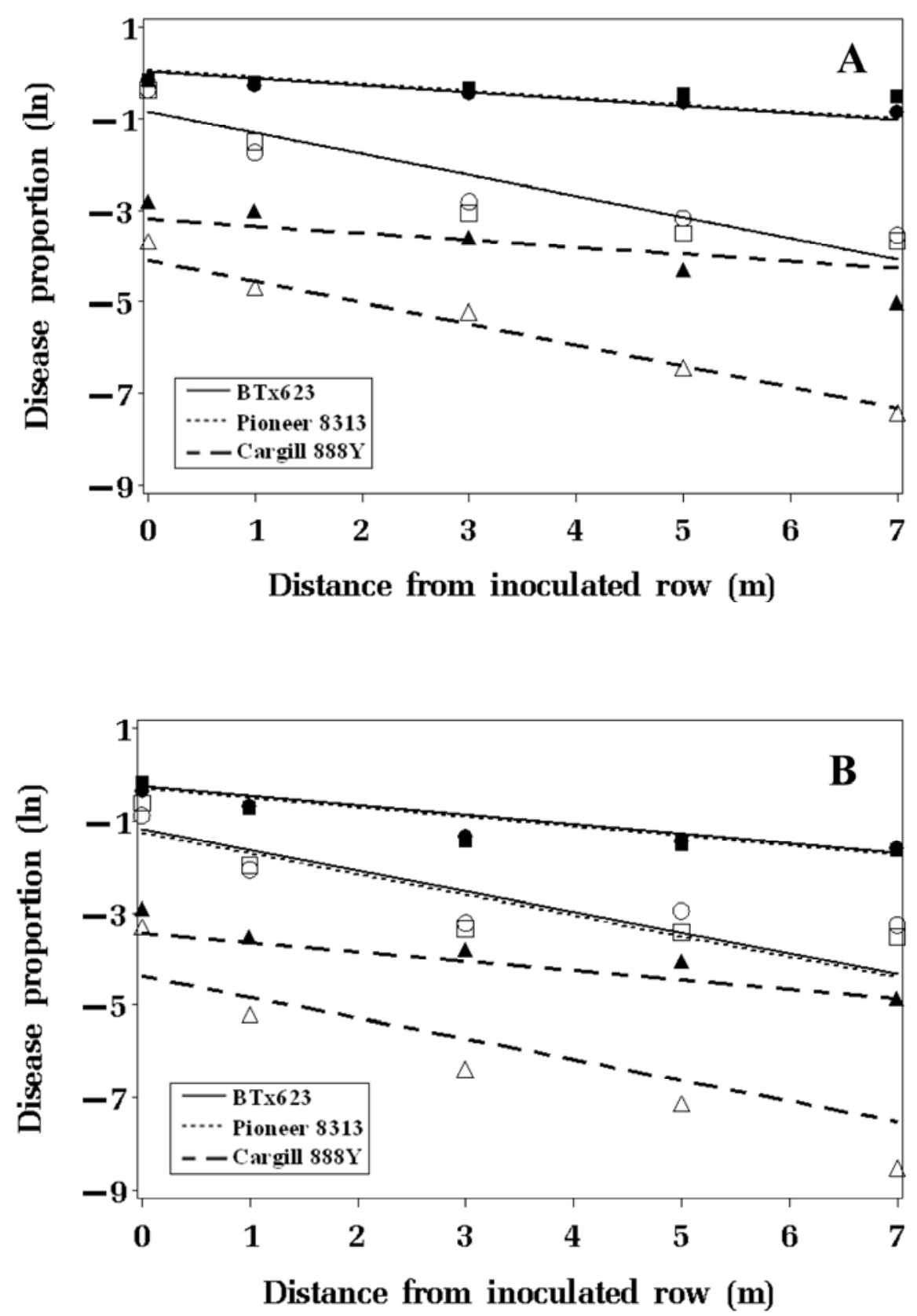

Fig. 2. Disease gradients of sorghum anthracnose on 'BTx623' (circle), 'Pioneer 8313' (square), and 'Cargill 888Y' (triangle) at 57 (open) and 78 (solid) days after inoculation in 2001 (A) and 2002 (B). Smooth lines represent the best fit of the negative linearized exponential model to the observed data. 
tive to upper asymptote of disease increase over time (34), the rate of disease increase will be given different meanings when $k$ values are different. Each analytical method is best suited to answer specific questions about the epidemics to be compared (3). Comparisons of infection rates between disease progress curves should be conducted under the situations with the results of the time of inflection point in the present study indicate that the incomparability of infection rates due to the big difference in $k$ may influence the value of $t_{i p}$

The results of comparisons of sAUDPC indicated that resistance in Cargill $888 \mathrm{Y}$ decreased the values of sAUDPC compared with susceptible BTx623 and Pioneer 8313. AUDPC values appeared to be more constant, reasonable, and easy to interpret since the AUDPC value incorporates the influence of the time of epidemic onset, rate of disease progress, and final disease severity $(27,33)$. AUDPC values have been widely used to evaluate disease resistance $(4,22,25)$. The advantage of AUDPC is that it can be used to compare and analyze disease epidemics that are not appropriately described using growth models.

This is the first detailed report on the quantitative analysis of disease gradients of sorghum anthracnose. Our results support a previous report that the negative exponential model outperformed the inverse power law model in fitting disease gradients of rain-splash diseases (4). However, disease intensity was underestimated at the inoculated rows and at $7 \mathrm{~m}$ from the source, and was overestimated in the middle parts of the observed distance. This may be due to the higher disease severities in the inoculated rows that resulted from higher inoculum pressures and more conidia dispersed on the same leaf or the same plant. Significantly lower slopes at 78 DAI than at 57 DAI may be explained by the secondary spread of disease and same or similar $k$ and $y_{0}$. The inconsistent estimated from $k, y_{0}$, and $r$.

increases in distance resulting from additional pathogen generations $(11,16)$, and also from restricted increases of disease severity at the source when the disease approached maximum in the negative exponential model (15). Fitt et al. (11) reported that half-distances ranged from 6 to $16 \mathrm{~cm}$ for splash-borne organisms in rain tower experiments. Pande et al. (23) observed that sorghum anthracnose spread from foci up to $9.75 \mathrm{~m}$ at physiological maturity. In the present study, disease severity on susceptible cultivars reached $65 \%$ at 7 $\mathrm{m}$ from the initial inoculum source. The half-distance estimated from the negative exponential model for sorghum anthracnose was about $1.5 \mathrm{~m}$ at 57 DAI and 3.3 to $4.5 \mathrm{~m}$ at $78 \mathrm{DAI}$ in the present study. Rainfall and wetness duration are critical factors for sorghum anthracnose development $(12,23)$ and sporulation on lesions (10). Nonetheless, use of overhead sprinkler irrigation in our experiments might have favored sporulation and dissemination of inoculum and caused the disease gradient to flatten more quickly.

Resistance to sorghum anthracnose in grain sorghum is considered to be nonspecific (2), although pathotypes of the pathogen are described by reaction to eight differential breeding lines (5). Using these criteria, Dale et al. (9) previously identified pathotype 4 in Arkansas. More recently, however, Moore et al. (19) reported that a number of new and highly aggressive pathotypes are now present in Arkansas virulent to grain sorghum. Additional research is needed to determine whether these strains and pathotypes develop as mixed populations on different grain sorghum hybrids and breeding lines or whether some strains and pathotypes become dominant during the development of an epidemic as described by Yang and TeBeest (32) on northern jointvetch.

\section{ACKNOWLEDGMENTS}

This research is a portion of a dissertation submitted by the senior author for the degree of Doctor of Philosophy in Plant Pathology at the University

Table 3. Comparisons of the intercepts $(\ln (a))$, gradient slope $(b)$, and half-distance $\left(d_{50 \%}\right)$ estimated from the linearized exponential models fitting disease gradients of sorghum anthracnose using the average disease proportion on three cultivars at two sampling dates in 2001 and 2002

\begin{tabular}{lclrcc}
\hline Year & DAI & Cultivar & \multicolumn{1}{c}{$\boldsymbol{\operatorname { l n } ( \boldsymbol { a } )}$} & $\boldsymbol{b}$ & $\boldsymbol{d}_{\mathbf{5 0} \boldsymbol{\%}}(\mathbf{m})$ \\
\hline 2001 & 57 & BTx623 & $-0.862 \mathrm{~b}^{\mathrm{z}}$ & $0.461 \mathrm{a}$ & $1.503 \mathrm{~b}$ \\
& & Pioneer 8313 & $-0.842 \mathrm{~b}$ & $0.461 \mathrm{a}$ & $1.503 \mathrm{~b}$ \\
& Cargill 888Y & $-4.101 \mathrm{~d}$ & $0.461 \mathrm{a}$ & $1.503 \mathrm{~b}$ \\
& \multirow{2}{*}{78} & BTx623 & $0.038 \mathrm{a}$ & $0.152 \mathrm{~b}$ & $4.560 \mathrm{a}$ \\
& & Pioneer 8313 & $0.058 \mathrm{a}$ & $0.152 \mathrm{~b}$ & $4.560 \mathrm{a}$ \\
& & Cargill 888Y & $-3.201 \mathrm{c}$ & $0.152 \mathrm{~b}$ & $4.560 \mathrm{a}$ \\
& \multirow{3}{*}{57} & BTx623 & $-1.194 \mathrm{~b}$ & $0.449 \mathrm{a}$ & $1.544 \mathrm{~b}$ \\
& & Pioneer 8313 & $-1.253 \mathrm{~b}$ & $0.449 \mathrm{a}$ & $1.544 \mathrm{~b}$ \\
& & Cargill 888Y & $-4.385 \mathrm{~d}$ & $0.449 \mathrm{a}$ & $1.544 \mathrm{~b}$ \\
& 78 & BTx623 & $-0.249 \mathrm{a}$ & $0.205 \mathrm{~b}$ & $3.381 \mathrm{a}$ \\
& & Pioneer 8313 & $-0.308 \mathrm{a}$ & $0.205 \mathrm{~b}$ & $3.381 \mathrm{a}$ \\
& & Cargill 888Y & $-3.440 \mathrm{c}$ & $0.205 \mathrm{~b}$ & $3.381 \mathrm{a}$ \\
\hline
\end{tabular}

y Days after inoculation.

${ }^{\mathrm{z}}$ Means followed by same letter within a column for each year were not significantly different from each other at $P=0.05$ according linear contrasts for $\ln (a)$ and $b$, and the two sample $t$ tests for $d_{50 \%}$. of Arkansas, Fayetteville. This research was supported in part by a grant from the Arkansas Corn and Grain Sorghum Research and Promotion Board to D. O. TeBeest and by the University of Arkansas Agricultural Experiment Station. Seeds of grain sorghum cultivars Pioneer 8313 and Cargill 888Y were kindly provided by the Pioneer Hi-Bred and Cargill Seed companies, respectively, to the Department of Plant Pathology at the University of Arkansas. Seeds of BTx623 were obtained from W. Rooney, Texas A\&M University. We thank E. Gbur in the Statistics Lab at the University of Arkansas for assistance in statistical analyses and J. Rupe in the Department of Plant Pathology at University Arkansas for his reviewing and helpful comments.

\section{LITERATURE CITED}

1. Ali, M. E. K., and Warren, H. L. 1992. Anthracnose of sorghum. Pages 203-208 in: Sorghum and Millets Diseases: A Second World Review. W. A. J. de Milliano, R. A. Frederiksen, and G. D. Bengston, eds. International Crops Research Institute for the Semi-Arid Tropics, Patancheru, India.

2. Boora, K. S., Frederiksen, R. A., and Magill, C. W. 1998. DNA-based markers for a recessive gene conferring anthracnose resistance in sorghum. Crop Sci. 38:1708-1709.

3. Campbell, C. L. 1998. Disease progress in time: Modeling and data analysis. Pages 181206 in: The Epidemiology of Plant Diseases. D. G. Jones, ed. Kluwer Academic Publisher, The Netherlands.

4. Campbell, C. L., and Madden, L. V. 1990. Introduction to Plant Disease Epidemiology. John Wiley \& Sons, New York.

5. Cardwell, K. F., Hepperly, P. R., and Frederiksen, R. A. 1989. Pathotypes of Colletotrichum graminicola and seed transmission of sorghum anthracnose. Plant Dis. 73:255-257.

6. Casela, C. R., and Frederiksen, R. A. 1993. Survival of Colletotrichum graminicola sclerotia in sorghum stalk residues. Plant Dis. 77:825-827.

7. Collins, S. D., Casela, C. R., and Frederiksen, R. A. 1992. Colletotrichum graminicola microsclerotia on sorghum debris as an overwintering inoculum source. Sorghum Newsl. $33: 25$.

8. Culbreath, A. K., Beute, M. K., and Campbell, C. L. 1991. Spatial and temporal aspects of epidemics of Cylindrocladium black rot in resistant and susceptible peanut genotypes. Phytopathology 81:144-150.

9. Dale, J. L., Kirkpatrick, T. L., and Cardwell, K. F. 1988. Sorghum anthracnose in southwest Arkansas. Arkansas Farm Res. 1988(4):17.

10. Ferreira, A. S., and Warren, H. L. 1982. Resistance of sorghum to Colletotrichum graminicola. Plant Dis. 66:773-775.

11. Fitt, B. D. L., Gregory, P. H., Todd, A. D., McCartney, H. A., and McDonald, O. C. 1987. Spore dispersal and plant disease gradients: A comparison between two empirical models. J. Phytopathol. 118:227-242.

12. Frederiksen, R. A. 2000. Leaf anthracnose Pages 10-12 in: Compendium of Sorghum Diseases. R. A. Frederiksen and G. N. Odvody, eds. American Phytopathological Society, St. Paul, MN.

13. Frederiksen, R. A., and Duncan, R. R. 1992. Sorghum disease in North America. Pages 8588 in: Sorghum and Millets Disease: A Second World Review. W. A. J. de Milliano, R. A Frederiksen, and C. D. Bengston, eds. International Crops Research Institute for the SemiArid Tropics, Patancheru, India.

14. Gilligan, C. A. 1990. Comparison of disease progress curves. New Phytol. 115:223-242.

15. Hess, D. E., Bandyopadhyay, R., and Sissoko, I. 2002. Pattern analysis of sorghum genotype $\times$ environment interaction for leaf, panicle, and grain anthracnose in Mali. Plant Dis. 86:13741382 . 
16. Maffia, L. A., and Berger, R. D. 1999. Models of plant disease epidemics. II: Gradients of bean rust. J. Phytopathol. 147:199-206.

17. Maiti, R. K. 1994. Sorghum Science. Science Publisher, Lebanon, NH, USA.

18. Minogue, K. P. 1986. Disease gradients and the spread of disease. Pages 285-310 in: Plant Disease Epidemiology Vol. 1: Population Dynamics and Management. K. J. Leonard and W. E. Fry, eds. Macmillan Publishing Company, New York.

19. Moore, J. W., Ditmore, M., and TeBeest, D. O. 2008. Pathotypes of Colletotrichum sublineolum in Arkansas. Plant Dis. 92:1415-1420.

20. Ngugi, H. K., Julian, A. M., King, S. B., and Peacocke, B. J. 2000. Epidemiology of sorghum anthracnose (Colletotrichum sublineolum) and leaf blight (Exserohilum turcicum) in Kenya. Plant Pathol. 49:129-140.

21. Ntahimpera, N., Dillard, H. R., Cobb, A. C., and Seem, R. C. 1996. Anthracnose development in mixtures of resistant and susceptible dry bean cultivars. Phytopathology 86:668-673.

22. Oña, I., Vera Gruz, C. M., Nelson, R. J., Leach, J. E., and Mew, T. W. 1998. Epidemic development of bacterial blight on rice carrying resistance genes $\mathrm{Xa}-4, \mathrm{Xa}-7$, and $\mathrm{Xa}-10$. Plant Dis. 82:1337-1340.

23. Pande, S., Thakur, R. P., Karunakar, R. I.,
Bandyopadhyay, R., and Reddy, B. V. S. 1994. Development of screening methods and identification of stable resistance to anthracnose in sorghum. Field Crops Res. 38:157-166.

24. Paysour, R. E., and Fry, W. E. 1983. Interplot interference: A model for planning field experiments with aerially disseminated pathogens. Phytopathology 73:1014-1020.

25. Pinkerton, J. N., Johnson, K. B., Aylor, D. E., and Stone, J. K. 2001. Spatial and temporal increase of eastern filbert blight in European hazelnut orchards in the Pacific Northwest. Phytopathology 91:1214-1223.

26. Prasad, K. V. V., Khare, M. N., and Jain, A. C. 1985. Site of infection and further development of Colletotrichum graminicola (Ces.) Wilson in naturally infected sorghum grains. Seed Sci. Technol. 13:37-40.

27. Reyrikds, K. L., and Cunfer, B. M. 1997. Components of partial host resistance and epidemic progress. Pages 111-114 in: Exercises in Plant Disease Epidemiology. L. J. Francl and D. A. Neher, eds. American Phytopathological Society, St. Paul, MN

28. Steinlage, T. A., Hill, J. H., and Nutter, F. W., Jr. 2002. Temporal and spatial spread of Soybean mosaic virus (SMV) in soybeans transformed with the coat protein gene of SMV. Phytopathology 92:478-486.
29. Thomas, M. D. 1992. Sorghum diseases in Western Africa. Pages 25-29 in: Sorghum and Millet Diseases: A Second Word Review. W. A J. de Milliano, R. A. Frederiksen, and G. D. Bengston, eds. International Crops Research Institute for the Semi-Arid Tropics, Patancheru, India.

30. Thomas, M. D., Sissoko, I., and Sacko, M. 1996. Development of leaf anthracnose and its effect on yield and grain weight of sorghum in West Africa. Plant Dis. 80:151-153.

31. Ware, S. W. 2001. Genetic diversity of Colletotrichum sublineolum based on vegetative compatibility, mtDNA and nuDNA restriction fragment length polymorphisms, and intron sequences. MS thesis. University of Arkansas, Fayetteville.

32. Yang, X. B., and TeBeest, D. O. 1995. Com petitiveness of mutant and wild-type isolates of Colletotrichum gloeosporioides f. sp. aes chynomene on northern jointvetch. Phytopathology 85:705-710.

33. Yu, D. Z., Yang, X. J., Yang, L. J., Jeger, M. J. and Brown, J. K. M. 2001. Assessment of partial resistance to powdery mildew in Chinese wheat varieties. Plant Breed. 120:279-284.

34. Zadoks, J. C., and Schein, R. D. 1979. Epidemiology and Plant Disease Management. Oxford University Press, New York. 